\title{
RETRACTED ARTICLE: Analysis of security operation and maintenance system using privacy utility in media environment
}

\author{
Zhengwei Jiang $^{1} \cdot$ Guoen Chen $^{2} \cdot$ Xueqi Jin ${ }^{1} \cdot$ \\ Yueqiang Wang ${ }^{2}$
}

Received: 11 October 2017 / Revised: 19 October 2017 / Accepted: 25 October 2017 / Published online: 1 February 2018

(C) Springer Science+Business Media, LLC 2018

The Editor-in-Chief has retracted this article [1], which was published as part of special issue "Multi-source Weak Data Management using Big Data", because there is evidence of figure duplication without appropriate permission, as well as evidence suggesting authorship manipulation and an attempt to subvert the peer review process.

The authors have not responded to correspondence about this retraction.

\section{References}

1. Jiang, Z., Chen, G., Jin, X. et al. Analysis of security operation and maintenance system using privacy utility in media environment. Multimed Tools Appl (2018). https://doi. org/10.1007/s11042-017-5361-y

Electronic supplementary material The online version of this article (https://doi.org/10.1007/s11042-0175361-y) contains supplementary material, which is available to authorized users.

Zhengwei Jiang

jiangzhengwei1778@126.com

1 State Grid Zhejiang Electric Power Co. Ltd, Hangzhou 310000, China

2 State Grid Jiaxing Electronic Power Supply Company, Jiaxing Shi, China 